\title{
A Case of Acute Necrotizing Pancreatitis Complicated by Portal Vein Thrombosis
}

Saurabh Kumar, Shantanu Kumar Sahu*, Jitendra P Ray, Sumit Jain, Vinamra Mittal, Kartik Nandra and Akshay Chauhan

Department of General Surgery, Himalayan Institute of Medical Sciences, Himalayan Institute Hospital Trust (HIHT) University, Swami Ram Nagar, Dehradun, India

\begin{abstract}
Portal vein thrombosis is an unusual complication of acute pancreatitis. It occurs in some cases of severe acute pancreatitis particularly those associated with pancreatic necrosis. A review of relevant literature suggests a role of anticoagulants in such cases but further studies are needed to establish the advantage of such therapy conclusively. We present a case of acute necrotizing pancreatitis complicated by portal and splenic vein thrombosis managed with anticoagulants. This resulted in recovery without development of portal hypertension and its complications.
\end{abstract}

Keywords: Acute necrotizing pancreatitis; Portal vein thrombosis

\section{Introduction}

Portal vein thrombosis is an unusual complication of acute pancreatitis. It occurs in some cases of severe acute pancreatitis particularly those associated with pancreatic necrosis. Role of anticoagulants in such cases is not well established [1]. We present a case of acute necrotizing pancreatitis complicated by portal and splenic vein thrombosis managed with anticoagulants which resulted in recovery without development of portal hypertension and its complications.

\section{Case Report}

A 20 years old boy presented to surgery emergency ward of our hospital with complaints of severe abdominal pain predominantly in epigastric region and repeated vomiting for last six days. He was being treated at a peripheral health centre so far but, as the symptoms became more severe he was referred to our hospital. There was no history of similar pain in past but he used to have some abdominal discomfort occasionally. He was a known alcoholic and used to consume alcohol in moderate amount regularly. On examination he was conscious and oriented. He had a pulse rate of $110 / \mathrm{min}$ and his blood pressure was 110/60 mm of Hg. A nasogastric tube and a Foley's catheter were already in place. After initial care he was subjected to laboratory and radiological investigation. He was having a total leukocyte count of $14,000 /$ microlitre. His serum amylase was $946 \mathrm{U} / \mathrm{L}$ and serum lipase was $262 \mathrm{U} / \mathrm{L}$. An X-ray of abdomen showed dilated bowel loops with few air-fluid levels and the X-ray chest showed mild left sided pleural effusion. An ultrasound of abdomen was done which did not reveal any significant abnormality. But in view of a strong clinical possibility of acute pancreatitis a contrast enhanced CT scan of abdomen was done. It revealed a bulky pancreas with multiple hypodense areas suggestive of pancreatic necrosis. Another striking feature was the presence of a thrombus in the portal vein and the splenic vein (Figure 1). The patient was shifted to intensive care unit and was managed conservatively. $\mathrm{He}$ was put on injection Meropenem one gram intravenously eight hourly. In view of portal vein thrombosis he was put on low molecular weight heparin for initial few days. The patient continued to have paralytic ileus for a few days with a persistent complaint of abdominal distension but had a smooth recovery thereafter. Once orally allowed he was switched to oral anticoagulants and was later on discharged from the hospital. Later on he developed a pseudocyst for cystogastrostomy was done. A follow up CT scan of abdomen showed the thrombus to have resolved and the patient did not develop features of portal hypertension.

\section{Discussion}

Acute pancreatitis has a variable clinical course regardless of its etiology. Most cases are self-limited. But approximately one quarter of patients develop vascular complications. The most common complications are hemorrhage into a pseudocyst, erosion of upper gastrointestinal arteries, thrombosis of the portal venous system, formation of varices or pseudoaneurysms and rupture of a pseudoaneurysm [1]. Splanchnic vein thrombosis is an unusual complication of acute pancreatitis and is particularly seen in association with necrotizing pancreatitis. It is rare in absence of pancreatic necrosis [2]. It may include thrombosis of one or more of splenic vein, portal vein and superior mesenteric vein. Isolated splenic vein thrombosis is relatively common but portal and superior mesenteric vein thrombosis are much less common [3]. In our case both splenic and portal veins were thrombosed.

Although splanchnic vein thrombosis is often an incidental finding on imaging in cases of acute necrotizing pancreatitis, its significance lies in the potentially fatal consequences. These include portal hypertension, and its complications especially upper GI bleed, small bowel ischaemia and liver failure. Although splanchnic vein thrombosis is assumed to arise usually in association with hypercoagulable state but in cases of acute pancreatitis the thrombosis of splanchnic veins probably occurs as a direct result of inflammation in the vicinity $[3,4]$.

The role of early institution of anticoagulation in such cases is controversial $[4,5]$. The European Network for Vascular Disorders of the Liver (EN-Vie) recommends the utilization of anticoagulation early in patients with acute PV thrombosis in non-cirrhotic, non-malignant patients [5]. The rate of recanalization is higher if anticoagulants are started earlier [3]. But the use of anticoagulants in this scenario

*Corresponding author: Shantanu Kumar Sahu, MS, FAIS, FMAS, FIAGES; Associate Professor, Department of General Surgery, Himalayan Institute of Medical Sciences, Swami Ram Nagar Post-Doiwala Dehradun Uttarakhand, India, Pin 248140, Tel: +91 (0) 94129338 68; Fax: +91 (0) 13524713 17; E-mail: Intshantanu@yahoo.co.in

Received February 19, 2014; Accepted March 20, 2014; Published September 20, 2014

Citation: Kumar S, Sahu SK, Ray JP, Jain S, Mittal V, et al. A Case of Acute Necrotizing Pancreatitis Complicated by Portal Vein Thrombosis. Journal of Surgery [Jurnalul de chirurgie] 2014; 10(2): 171-172 DOI: 10.7438/1584-9341-10-2-13

Copyright: $\odot 2014$ Kumar S, et al. This is an open-access article distributed under the terms of the Creative Commons Attribution License, which permits unrestricted use, distribution, and reproduction in any medium, provided the original author and source are credited. 


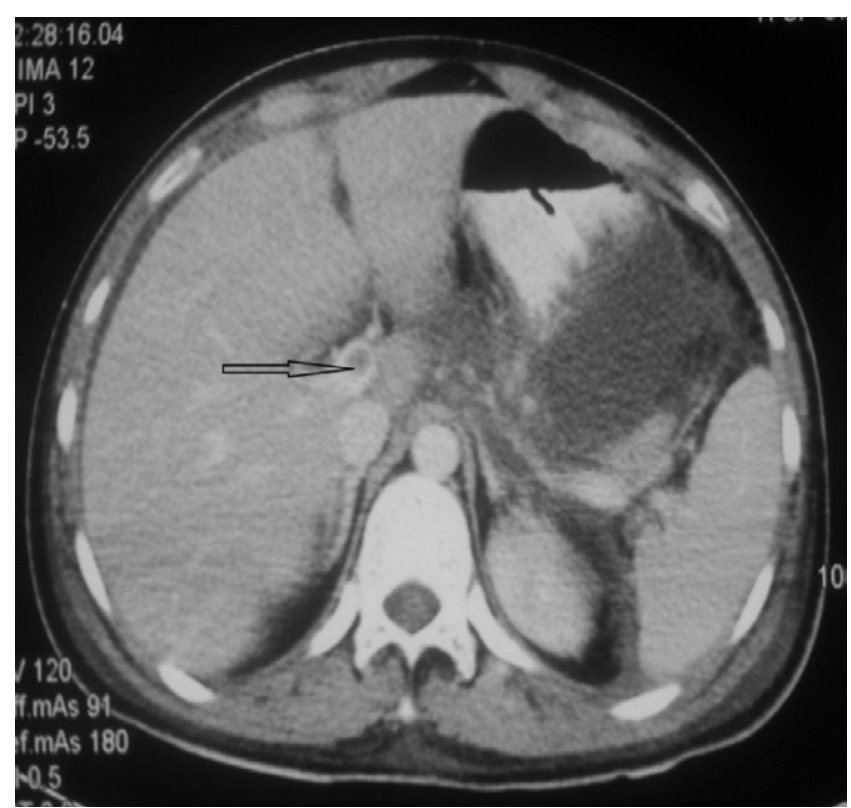

Figure 1: CECT image of abdomen showing portal vein thrombosis with acute pancreatitis.

poses challenge as these patients are at increased risk of hemorrhage because of pseudoaneurysms and the need for surgical interventions for management of pancreatic necrosis and abscess [4]. Nevertheless available evidence favors the use of anticoagulants and we used first the low molecular weight heparin followed by oral anticoagulant for three months. Review CT scan done three months later showed recanalization of portal vein and although the patient developed a pseudocyst he, at no point of time, showed any evidence of portal hypertension or development of varices. But certainly randomized controlled trials are needed to establish the role of anticoagulants in such cases.

\section{Conclusions}

Splanchnic vein thrombosis is an unusual complication of acute pancreatitis and is seen particularly in cases complicated by pancreatic necrosis. Although in our case institution of anticoagulants resulted in recovery without development of portal hypertension, randomized trials are required to establish the exact role of such therapy in case of splanchnic vein thrombosis.

\section{Conflict of Interests}

Authors have no conflict of interests to disclose.

\section{References}

1. Mallick IH, Winslet MC (2004) Vascular complications of pancreatitis. JOP 5 : 328-337.

2. Butler JR, Eckert GJ, Zyromski NJ, Leonardi MJ, Lillemoe KD, et al. (2011) Natural history of pancreatitis-induced splenic vein thrombosis: a systematic review and meta-analysis of its incidence and rate of gastrointestinal bleeding. HPB (Oxford) 13: 839-845.

3. Park WS, Kim HI, Jeon BJ, Kim SH, Lee SO (2012) Should anticoagulants be administered for portal vein thrombosis associated with acute pancreatitis? World J Gastroenterol 18: 6168-6171.

4. Gonzelez HJ, Sahay SJ, Samadi B, Davidson BR, Rahman SH (2011) Splanchnic vein thrombosis in severe acute pancreatitis: a 2-year, singleinstitution experience. HPB (Oxford) 13: 860-864.

5. Plessier A, Darwish-Murad S, Hernandez-Guerra M, Consigny $Y$, Fabris F, et al. (2010) Acute portal vein thrombosis unrelated to cirrhosis: a prospective multicenter follow-up study. Hepatology 51: 210-218. 